\title{
A Perturbation Analysis of Transient Freezing of a Laminar Liquid Flow in a Cooled Two- Dimensional Channel
}

\begin{abstract}
H. Beer
Technische Hochschule Darmstadt. Institut für Technische Thermodynamik, 6100 Darmstadt, Federal Republic of
\end{abstract} Germany

\begin{abstract}
The paper shows the applicability of a regular perturbation method for predicting the transient development of the ice layer thickness inside a cooled planar channel subjected to laminar flow. Applying the perturbation expansion to the conservation equations, closed-form solutions for the velocity and temperature distributions in the fluid for an arbitrarily shaped channel could be derived under the assumption that the axial variation in solid layer thickness is small. The distributions obtained for the steady-state ice layer thickness and the velocity were checked by numerical calculations and compared with the measurements of Kikuchi et al. (1986) and a generally good agreement was found.
\end{abstract}

\section{Introduction}

Problems of solidification or freezing of liquids inside cold channels have been encountered in numerous engineering applications. Because the freezing shut of systems may lead to a destruction of the equipment (for example, freezing shut of a water pipe in winter or freezing of molten sodium in a nuclear reactor), it is advisable to prevent blockage. If solidification on the cooled walls cannot be suppressed, steady-state conditions must be sought.

Many theoretical and experimental studies have been performed for fluid flow with solidification in circular tubes. An early investigation was reported by Zerkle and Sunderland (1968) for the steady-state freezing of laminar flow inside a horizontal tube. Under the assumption of a parabolic axial velocity distribution throughout the full axial length of the tube and with an appropriate coordinate transform, they were able to reduce the problem to the classical Graetz problem without solidification. Özișik and Mulligan (1969) used a slug flow approximation for the liquid core to analyze transient freezing in an isothermal circular tube. They applied integral transforms to obtain the transient development of the ice layer inside the tube. Bilenas and Jiji (1970) solved the boundary layer equations applying a finite-difference scheme, but they used a wide-meshed grid for their calculations. Chida (1983) calculated numerically the steady-state ice layer thickness, under consideration of axial conduction. Bilenas and Jiji (1970) and Chida (1983) assumed a fully developed parabolic axial velocity distribution at the entrance of the cooled section.

Despite its relevance to any important technological and physical problems, the freezing of liquid flows through a cooled two-dimensional channel has not been studied as intensively as the freezing in a cooled circular pipe. An early investigation of this problem was reported by Lee and Zerkle (1969). They assumed the axial velocity to be parabolic throughout the whole chill region, which was in analogy to Zerkle and Sunderland (1968). With the approximated velocity profile and with an appropriate coordinate transform, the energy equation could be reduced to a Graetz problem, and the steady-state ice layer was calculated. An experimental investigation of the effect of freezing a liquid in case of laminar flow between two cooled plates has been performed only by Kikuchi et al. (1986). They

Contributed by the Heat Transfer Division for publication in the JourNal of Heat Transfer. Manuscript received by the Heat Transfer Division February 1992; revision received September 1992. Keywords: Forced Convection, PhaseChange Phenomena, Transient and Unsteady Heat Transfer. Associate Technical Editor: Y. Bayazitoglu. used water as the working fluid. Both plates were maintained at the same temperature, which was below the freezing temperature of the water and varied from $-2^{\circ} \mathrm{C}$ to $-7^{\circ} \mathrm{C}$. A numerical calculation of the steady-state ice layers for an arbitrary velocity profile at the entrance of the chill region was given by Weigand and Beer (1991), who solved the boundary layer equations with the help of a finite-difference method. For the case of a fully developed parabolic velocity distribution at the entrance of the cooled channel, the results of Weigand and Beer (1991) were compared with the experimental findings of Kikuchi et al. (1986) and a generally good agreement was found. Bennon and Incropera (1988) studied numerically the influence of free convection effects on the axial distribution of the steady-state solid-liquid interface by solving the conservation equations for laminar flow without simplified assumptions. The transient development of the solidified crust in a planar channel has been studied only by Weigand and Beer (1992). They obtained an approximate analytical solution for the distribution of the ice layer thickness under the assumption that the axial variation in solid layer thickness could be neglected. Their analysis is based on the boundary layer equations.

The subject of this paper is the presentation of an approximate solution of the boundary layer equations with the help of a regular perturbation method. Under the assumption that the axial variation in solid layer thickness is small, closed-form solutions for the velocity and temperature distributions in the fluid for an arbitrarily shaped channel could be developed. The given method is quite flexible and can be used to calculate the velocity and temperature distributions in a channel with an arbitrary cross section, if the axial variation in the free channel height is comparatively small. Finally, it can be shown that the solutions of Zerkle and Sunderland (1969) and Weigand and Beer (1992) can be obtained as the zero-order solution of the regular perturbation expansion, presented here.

Moreover, it should be pointed out that the present paper deals solely with smooth ice layers in laminar liquid flow. This means that the ice layers are assumed to increase monotonously in thickness with increasing values of the axial coordinate.

\section{Analysis}

Basic Equations and Assumptions. Figure 1 shows the geometric configuration and the coordinate system for a planar symmetric channel. The fluid enters the chilled region at $x=0$ with a fully developed laminar velocity profile and with a 


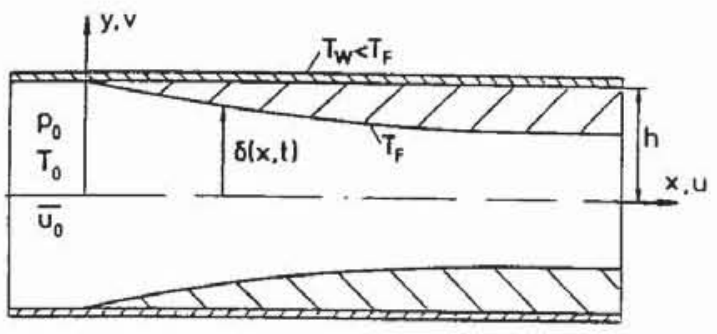

Fig. 1 Physical model and coordinate system

constant temperature $T_{0}$. In the cooled section, the wall temperature is maintained at a constant value $T_{W}$, which is lower than the freezing temperature $T_{F}$ of the fluid. The frozen layers are generated on both walls as the fluid proceeds along the channel. Assuming an incompressible, Newtonian fluid with constant fluid properties, the quasi-steady conservation equations for the fluid may be written in the following form:

$$
\begin{gathered}
\frac{\partial \tilde{u}}{\partial \tilde{x}}+\frac{\partial \tilde{v}}{\partial \tilde{y}}=0 \\
\tilde{u} \frac{\partial \tilde{u}}{\partial \tilde{x}}+\tilde{v} \frac{\partial \tilde{u}}{\partial \tilde{y}}=-\frac{\partial \tilde{p}}{\partial \tilde{x}}+\frac{1}{\operatorname{Re}_{h}} \frac{\partial^{2} \tilde{u}}{\partial \tilde{y}^{2}} \\
0=\frac{\partial \tilde{p}}{\partial \tilde{y}} \\
\tilde{u} \frac{\partial \theta}{\partial \tilde{x}}+\tilde{v} \frac{\partial \theta}{\partial \tilde{y}}=\frac{1}{\operatorname{Re}_{h} \operatorname{Pr}} \frac{\partial^{2} \theta}{\partial \tilde{y}^{2}}
\end{gathered}
$$

with the dimensionless quantities

$$
\begin{aligned}
\tilde{x}=\frac{x}{h}, \quad \tilde{y}=\frac{y}{h}, \quad \tilde{u} & =\frac{u}{\bar{u}_{0}}, \quad \tilde{v}=\frac{v}{\bar{u}_{0}}, \quad \tilde{p}=\frac{p}{p \bar{u}_{0}^{2}}, \tilde{\delta}=\frac{\delta}{h} \\
\theta & =\frac{T-T_{F}}{T_{0}-T_{F}}, \quad \operatorname{Re}_{h}=\frac{\bar{u}_{0} h}{\nu}, \quad \operatorname{Pr}=\frac{\nu}{a}
\end{aligned}
$$

where $\bar{u}_{0}$ denotes the axial mean velocity for $\tilde{x}=0$. The boundary conditions belonging to Eqs. (1)-(4) are

$$
\begin{gathered}
\tilde{x}=0: \tilde{u}=\frac{3}{2}\left(1-\tilde{y}^{2}\right), \quad \tilde{p}=\tilde{p}_{0}, \quad \theta=1 \\
\tilde{y}=0: \frac{\partial \tilde{u}}{\partial \tilde{y}}=0, \quad \tilde{v}=0, \quad \frac{\partial \theta}{\partial \tilde{y}}=0 \\
\tilde{y}=\bar{\delta}: \tilde{u}=0, \quad \tilde{v}=0, \quad \theta=0
\end{gathered}
$$

By deriving Eqs. (1)-(4), the usual boundary-layer assumptions were made, which are a common treatment of the conservation equations for channel flows (Cebeci and Chang, 1978). The assumption of quasi-steady conditions, which was incorporated into Eqs. (1)-(5), is justified for water flow because of the small value of the Stefan number, which is about Ste $\approx 0.1$ (Cervantes et al., 1990). The conservation of mass in integral form is given by

$$
1=\int_{0}^{\tilde{\delta}} \tilde{u} d \tilde{y}
$$

where the mass that gets lost by freezing at the channel walls was neglected as the change in the density between fluid and solid is very small for water and quasi-steady conditions are assumed. As a consequence of these assumptions, the resulting velocity at the freezing front in Eq. (6) is taken to be zero.

In addition to Eqs. (1)-(7), the energy equation for the solid region is required. Assuming constant properties in the solid region and negligible axial conduction, the heat conduction equation for the solid phase reduces for quasi-steady conditions to

$$
\frac{\partial^{2} \theta_{S}}{\partial \tilde{y}^{2}}=0
$$

with the boundary conditions

$$
\begin{aligned}
& \tilde{y}=\tilde{\delta}: \theta_{S}=1 \\
& \tilde{y}=1: \theta_{S}=0
\end{aligned}
$$

The dimensionless temperature $\theta_{S}$ is defined as $\theta_{S}=\left(T-T_{W}\right) /$ $\left(T_{F}-T_{W}\right)$. Equations (1)-(6) and Eqs. (8)-(9) are coupled by the interface energy equation

$$
\frac{\partial \theta_{S}}{\partial \tilde{y}}-\frac{1}{B} \frac{\partial \theta}{\partial \tilde{y}}=\frac{\partial \tilde{\delta}}{\partial \tau}\left[1+\left(\frac{\partial \tilde{\delta}}{\partial \tilde{x}}\right)^{2}\right]^{-1}, \quad \tilde{y}=\tilde{\delta}
$$

with the following dimensionless quantities:

$$
\begin{aligned}
& B=\frac{k_{s}}{k} \frac{T_{F}-T_{W}}{T_{0}-T_{F}}, \quad \tau=\text { FoSte, } \quad \text { Fo }=\frac{t a_{S}}{h^{2}}, \\
& \qquad \text { Ste }=\frac{c_{S}\left(T_{F}-T_{W}\right)}{r_{S}}
\end{aligned}
$$

where $B$ denotes a dimensionless freezing parameter.

Velocity and Temperature Distribution in the Liquid Phase. The velocity and the temperature distribution in the liquid can be calculated from Eqs. (1)-(7). Introducing a streamfunction, defined by

$$
\tilde{u}=\frac{\partial \psi}{\partial \tilde{y}}, \quad \tilde{v}=-\frac{\partial \psi}{\partial \tilde{x}}
$$

into Eqs. (1)-(7) and applying the coordinate transformation

$$
\eta=\frac{\tilde{y}}{\tilde{\delta}}, \quad \xi=\frac{1}{\operatorname{Re}_{h}} \int_{0}^{\bar{x}} \frac{d \hat{x}}{\bar{\delta}}
$$

\section{Nomenclature}

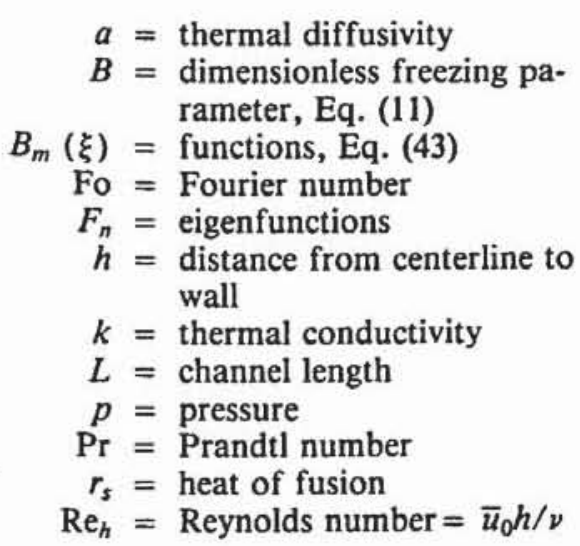

$a=$ thermal diffusivity rameter, Eq. (11)

$(\xi)=$ functions, Eq. (43)

Fo $=$ Fourier number

$F_{n}=$ eigenfunctions wall

$k=$ thermal conductivity

$L=$ channel length

$p=$ pressure

$r_{s}=$ heat of fusion

$\operatorname{Re}_{h}=$ Reynolds number $=\bar{u}_{0} h / \nu$

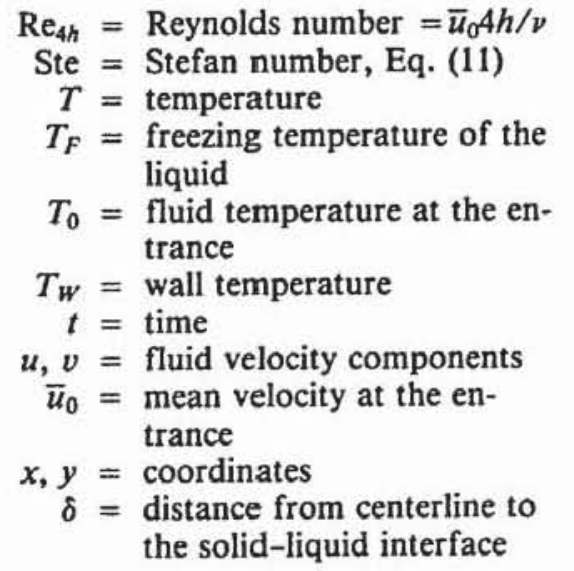

$\eta=$ modified vertical coordinate, Eq. (13)

$\theta=$ dimensionless temperature of the fluid $=\left(T-T_{F}\right) /$ $\left(T_{0}-T_{F}\right)$

$\theta_{S}=$ dimensionless temperature of the solid $=\left(T-T_{w}\right) /$ $\left(T_{F}-T_{W}\right)$

$\lambda_{n}=$ eigenvalues

$\nu=$ kinematic viscosity

$\xi=$ integral coordinate, Eq. (13)

$\rho=$ density

$\tau=$ dimensionless time

$\psi=$ streamfunction 
to the resulting equations, the following set of partial differential equations can be derived:

$$
\begin{gathered}
\frac{\partial \psi}{\partial \eta} \frac{\partial^{2} \psi}{\partial \xi \partial \eta}-\frac{\partial \psi}{\partial \xi} \frac{\partial^{2} \psi}{\partial \eta^{2}}-\frac{1}{\bar{\delta}} \frac{\partial \bar{\delta}}{\partial \xi}\left(\frac{\partial \psi}{\partial \eta}\right)^{2}=-\bar{\delta}^{2} \frac{\partial \tilde{p}}{\partial \xi}+\frac{\partial^{3} \psi}{\partial \eta^{3}} \\
0=\frac{\partial \tilde{p}}{\partial \eta} \\
\frac{\partial \psi}{\partial \eta} \frac{\partial \theta}{\partial \xi}-\frac{\partial \psi}{\partial \xi} \frac{\partial \theta}{\partial \eta}=\frac{1}{\operatorname{Pr}} \frac{\partial^{2} \theta}{\partial \eta^{2}}
\end{gathered}
$$

The boundary conditions, according to Eq. (6), are given by

$$
\begin{gathered}
\xi=0: \psi=\frac{3}{2}\left(\eta-\frac{1}{3} \eta^{3}\right)-1, \quad \tilde{p}=\tilde{p}_{0}, \quad \theta=1 \\
\eta=0: \quad \frac{\partial^{2} \psi}{\partial \eta^{2}}=0, \quad \psi=-1, \quad \frac{\partial \theta}{\partial \eta}=0 \\
\eta=1: \frac{\partial \psi}{\partial \eta}=0, \quad \psi=0, \quad \theta=0
\end{gathered}
$$

Without loss of generality, the streamfunction was assumed to be zero for $\eta=1$. The evaluation of the conservation of mass in integral form leads to $\psi=-1$ for $\eta=0$. In Eq. (17) the inlet boundary condition for $\tilde{u}$ was replaced by a condition for the stream function at $\xi=0$.

The coordinate transform, according to Eq. (13), is very useful in order to solve the conservation equations, because the duct with variable distance between the wall and the centerline is transformed into a duct with constant height.

Equation (15) states that the pressure is not a function of the coordinate $\eta$. Therefore, one obtains the following partial differential equation for $\psi$ by differentiating Eq. (14) with respect to $\eta$

$$
\frac{\partial \psi}{\partial \eta} \frac{\partial^{3} \psi}{\partial \xi \partial \eta^{2}}-\frac{\partial \psi}{\partial \xi} \frac{\partial^{3} \psi}{\partial \eta^{3}}=\frac{\partial^{4} \psi}{\partial \eta^{4}}+\frac{2}{\delta} \frac{\partial \bar{\delta}}{\partial \xi} \frac{\partial \psi}{\partial \eta} \frac{\partial^{2} \psi}{\partial \eta^{2}}
$$

The last term on the right-hand side of Eq. (18) is of the order $(1 / \delta)(\partial \bar{\delta} / \partial \xi)$. This term represents the effect of acceleration due to the converging ice layers. For moderate values of the cooling parameter $B$ this term is relatively small. Therefore, this quantity will be treated as a perturbation parameter. Let us assume an expansion for the streamfunction $\psi$ and also for the temperature distribution $\theta$ of the form

$$
\begin{gathered}
\psi=\psi_{0}+\frac{1}{\tilde{\delta}} \frac{\partial \tilde{\delta}}{\partial \xi} \psi_{1}+\left(\frac{1}{\bar{\delta}} \frac{\partial \tilde{\delta}}{\partial \xi}\right)^{2} \psi_{2}+\frac{1}{\bar{\delta}} \frac{\partial^{2} \tilde{\delta}}{\partial \xi^{2}} \psi_{3}+\ldots \\
\theta=\theta_{0}+\frac{1}{\bar{\delta}} \frac{\partial \bar{\delta}}{\partial \xi} \theta_{1}+\left(\frac{1}{\bar{\delta}} \frac{\partial \tilde{\delta}}{\partial \xi}\right)^{2} \theta_{2}+\frac{1}{\bar{\delta}} \frac{\partial^{2} \tilde{\delta}}{\partial \xi^{2}} \theta_{3}+\ldots
\end{gathered}
$$

whereby the perturbation quantity is a function of $\xi$ for a fixed value of time. Inserting the expansions, according to Eqs. (19) and (20), into the conservation Eqs. (16), (18) and into the boundary conditions (17), results in the following set of partial differential equations:

\section{Zero-Order Equations ( 1).}

$$
\begin{aligned}
& \frac{\partial \psi_{0}}{\partial \eta} \frac{\partial^{3} \psi_{0}}{\partial \xi \partial \eta^{2}}-\frac{\partial \psi_{0}}{\partial \xi} \frac{\partial^{3} \psi_{0}}{\partial \eta^{3}}=\frac{\partial^{4} \psi_{0}}{\partial \eta^{4}} \\
& \frac{\partial \psi_{0}}{\partial \eta} \frac{\partial \theta_{0}}{\partial \xi}-\frac{\partial \psi_{0}}{\partial \xi} \frac{\partial \theta_{0}}{\partial \eta}=\frac{1}{\operatorname{Pr}} \frac{\partial^{2} \theta_{0}}{\partial \eta^{2}}
\end{aligned}
$$

with the boundary conditions

$$
\begin{aligned}
& \xi=0: \psi_{0}=\frac{3}{2}\left(\eta-\frac{1}{3} \eta^{3}\right)-1, \quad \theta_{0}=1 \\
& \eta=0: \psi_{0}=-1, \quad \frac{\partial^{2} \psi_{0}}{\partial \eta^{2}}=0, \quad \frac{\partial \theta_{0}}{\partial \eta}=0
\end{aligned}
$$

$$
\eta=1: \psi_{0}=0, \quad \frac{\partial \psi_{0}}{\partial \eta}=0, \quad \theta_{0}=0
$$

First-Order Equations $(\sim(1 / \tilde{\delta})(\partial \tilde{\delta} / \partial \xi))$.

$$
\begin{gathered}
\frac{\partial \psi_{0}}{\partial \eta} \frac{\partial^{3} \psi_{1}}{\partial \xi \partial \eta^{2}}+\frac{\partial \psi_{1}}{\partial \eta} \frac{\partial^{3} \psi_{0}}{\partial \xi \partial \eta^{2}}-\frac{\partial \psi_{1}}{\partial \xi} \frac{\partial^{3} \psi_{0}}{\partial \eta^{3}}-\frac{\partial \psi_{0}}{\partial \xi} \frac{\partial^{3} \psi_{1}}{\partial \eta^{3}} \\
=\frac{\partial^{4} \psi_{1}}{\partial \eta^{4}}+2 \frac{\partial \psi_{0}}{\partial \eta} \frac{\partial^{2} \psi_{0}}{\partial \eta^{2}} \\
\frac{\partial \psi_{0}}{\partial \eta} \frac{\partial \theta_{1}}{\partial \xi}-\frac{\partial \psi_{0}}{\partial \xi} \frac{\partial \theta_{1}}{\partial \eta}=\frac{1}{\operatorname{Pr}} \frac{\partial^{2} \theta_{1}}{\partial \eta^{2}}-\frac{\partial \psi_{1}}{\partial \eta} \frac{\partial \theta_{0}}{\partial \xi}+\frac{\partial \theta_{0}}{\partial \eta} \frac{\partial \psi_{1}}{\partial \xi}
\end{gathered}
$$

with the homogeneous boundary conditions

$$
\begin{gathered}
\xi=0: \psi_{1}=0, \quad \theta_{1}=0 \\
\eta=0: \psi_{1}=0, \quad \frac{\partial^{2} \psi_{1}}{\partial \eta^{2}}=0, \quad \frac{\partial \theta_{1}}{\partial \eta}=0 \\
\eta=1: \psi_{1}=0, \quad \frac{\partial \psi_{1}}{\partial \eta}=0, \quad \theta_{1}=0
\end{gathered}
$$

It should be noted that only the zero-order and first-order problem are given here. This is done in view of the fact that only these two problems will be solved subsequently. The extrapolation to higher-order problems is straightforward, because the perturbation equations can be solved in succession.

The zero-order equation, Eq. (21), for the streamfunction, with the boundary conditions given by Eq. (23), can easily be solved. The resulting expression for $\psi_{0}$ is

$$
\psi_{0}=\frac{3}{2}\left(\eta-\frac{1}{3} \eta^{3}\right)-1
$$

Equation (27) states the fact that the axial velocity profile is parabolic through the whole chill region. After inserting the expression for the streamfunction $\psi_{0}$ into the energy Eq. (22), the following Graetz problem will be obtained:

$$
\frac{3}{2}\left(1-\eta^{2}\right) \frac{\partial \theta_{0}}{\partial \xi}=\frac{1}{\operatorname{Pr}} \frac{\partial^{2} \theta_{0}}{\partial \eta^{2}}
$$

Equation (28) can be solved easily by applying the method of separation of variables to $\theta_{0}$ in the form

$$
\theta_{0}=F(\eta) H(\xi)
$$

The temperature distribution $\theta_{0}$, which satisfies Eq. (28) and the given boundary conditions according to Eq. (23), is found to be

$$
\theta_{0}=\sum_{n=1}^{\infty} A_{n} F_{n}(\eta) \exp \left(-\frac{2}{3} \frac{\xi}{\operatorname{Pr}} \lambda_{n}^{2}\right)
$$

The eigenvalues $\lambda_{n}^{2}$ and the constants $A_{n}$ are given for example by Shah and London (1978). It is interesting to note that the zero-order solution for the velocity and temperature distributions in the fluid represents those given by Lee and Zerkle (1969) for steady-state conditions. Hence, it can be concluded that Lee and Zerkle (1969) ignored in their analysis the effect of acceleration due to converging ice layers on the velocity and temperature distributions in the fluid and calculated the thermal development of a hydrodynamically fully developed flow in the transformed $(\xi, \eta)$ plane.

Inserting the expression for the streamfunction $\psi_{0}$, Eq. (27), into Eq. (24), results in the following linear partial differential equation for $\psi_{1}$ :

$$
\frac{3}{2}\left(1-\eta^{2}\right) \frac{\partial^{3} \psi_{1}}{\partial \xi \partial \eta^{2}}+3 \frac{\partial \psi_{1}}{\partial \xi}=\frac{\partial^{4} \psi_{1}}{\partial \eta^{4}}-9 \eta\left(1-\eta^{2}\right)
$$

with the boundary conditions given by Eq. (26). Equation (31) can be solved with the aid of the Laplace transform

$$
L\left\{\psi_{1}(\xi, \eta)\right\}=\int_{0}^{\infty} \psi_{1}(\xi, \eta) \exp (-s \xi) d \xi \equiv \bar{\psi}_{1}(s, \eta)
$$


with respect to the axial coordinate $\xi$. This results in an ordinary differential equation for the transformed function $\bar{\psi}_{1}$

$$
\frac{3}{2}\left(1-\eta^{2}\right) s \frac{d^{2} \bar{\psi}_{1}}{d \eta^{2}}+3 s \bar{\psi}_{1}=\frac{d^{4} \bar{\psi}_{1}}{d \eta^{4}}+\frac{9}{s}\left(\eta^{3}-\eta\right)
$$

with the transformed boundary conditions

$$
\begin{aligned}
& \eta=0: \bar{\psi}_{1}=0, \quad \frac{d^{2} \bar{\psi}_{1}}{d \eta^{2}}=0 \\
& \eta=1: \bar{\psi}_{1}=0, \quad \frac{d \bar{\psi}_{1}}{d \eta}=0
\end{aligned}
$$

Equation (33) can be solved by assuming a series solution for $\bar{\psi}_{1}$ of the form

$$
\bar{\psi}_{1}=\sum_{v=1}^{\infty} a_{2 v-1} \eta^{2 v-1}+\sum_{v=1}^{\infty} a_{2 v} \eta^{2 v}
$$

Inserting the above given power series for $\bar{\psi}_{1}$ into the differential Eq. (33) and into the boundary conditions (34), it can easily be shown that the coefficients $a_{2,}$ for the even-powered terms are identically zero. Therefore, the first sum appearing in Eq. (35) represents the complete solution for $\bar{\psi}_{1}$ :

$$
\bar{\psi}_{1}=\sum_{v=1}^{\infty} a_{2 v-1} \eta^{2 v-1}
$$

It is obvious that Eq. (36) automatically satisfies the boundary conditions on $\bar{\psi}_{1}$ for $\eta=0$. Using expression (36) for $\bar{\psi}_{1}$ in Eqs. (33) and (34) results in a system of infinite coupled linear algebraic equations for the unknown $a_{2 \nu-1}$.

The power series solutions for $\bar{\psi}_{1}$ converges very rapidly with an increasing number of terms for arbitrary values of $s$. Therefore, only a five-term expansion was used to approximate the distribution of $\bar{\psi}_{1}$. After calculating the coefficients $a_{1}, \ldots, a_{9}$, a rational function for $\bar{\psi}_{1}$ of $s$ and $\eta$ is obtained. Using partial fraction expansions, $\bar{\psi}_{1}$ can be written as

$$
\bar{\psi}_{1}=\frac{A(\eta)}{s+b}+\frac{B(\eta) s+C(\eta)}{(s+a)^{2}+\omega^{2}}+\frac{1}{s} f_{2}(\eta)
$$

with the following abbreviations:

$$
\begin{gathered}
f_{0}(\eta)=\frac{1}{149}\left(-840 \eta^{9}+3312 \eta^{7}-7056 \eta^{5}+7536 \eta^{3}-2952 \eta\right) \\
f_{1}(\eta)=\frac{1}{5215}\left(1575 \eta^{9}+1302 \eta^{7}-8379 \eta^{5}+6552 \eta^{3}-1050 \eta\right) \\
f_{2}(\eta)=\frac{1}{840}\left(-9 \eta^{7}+63 \eta^{5}-99 \eta^{3}+45 \eta\right) \\
A(\eta)=\frac{f_{0}(\eta)-b f_{1}(\eta)-b^{2} f_{2}(\eta)}{\omega^{2}+(a-b)^{2}} \\
B(\eta)=-\left(f_{2}(\eta)+A(\eta)\right) \\
C(\eta)=-\frac{\omega^{2}+a^{2}}{b} A(\eta)-f_{0}(\eta)
\end{gathered}
$$

and the constants

$$
a=5.04390800, b=11.80480144, \omega=22.57064595
$$

The inverse Laplace transformation $\bar{\psi}_{1}$, according to Eq. (37), can be obtained directly from existing tables of transforms (see, e.g., Andrews and Shivamoggi, 1988). This results in

$\psi_{1}(\xi, \eta)=A(\eta) \exp (-b \xi)+\exp (-a \xi)$

$$
\begin{aligned}
\times[B(\eta)(\cos (\omega \xi) & \left.-\frac{a}{\omega} \sin (\omega \xi)\right) \\
& \left.+\frac{C(\eta)}{\omega} \sin (\omega \xi)\right]+f_{2}(\eta)
\end{aligned}
$$

The functions $f_{0}(\eta), f_{1}(\eta)$, and $f_{2}(\eta)$ satisfy independently the boundary conditions for $\eta=0$ and $\eta=1$, according to Eq. (26).

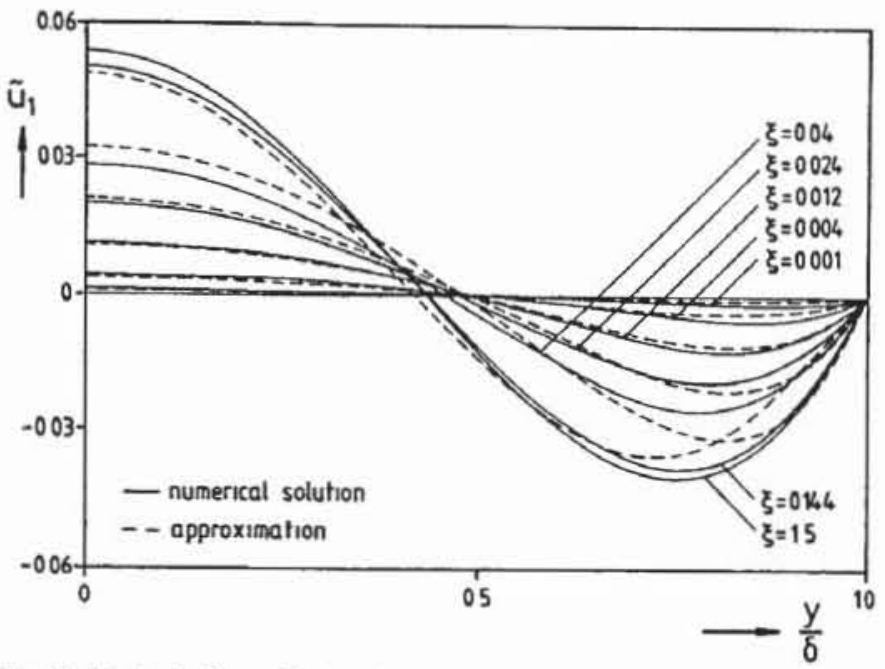

Fig. 2 Perturbation velocity $\tilde{u}_{1}$ as a function of $\pi$ for various values of the axial coordinate $\xi$

The function $f_{2}(\eta)$, which appears as the last term on the righthand side of Eq. (40), represents the solution of Eq. (31) for large values of $\xi$. Further, it is interesting to note that the monotonously decreasing part of the solution $A(\eta) \exp (-b \xi)$ tends more rapidly to zero for increasing values of $\xi$ than the second term on the right-hand side Eq. (40), which contains oscillating terms in $\xi$. In order to check the accuracy of the given solution for $\psi_{1}$, the linear partial differential Eq. (31), with the boundary conditions (26), was solved numerically with a finite-difference method. The applied implicit finite-difference scheme was the Keller-box method (Cebeci and Bradshaw, 1984). This method has several very desirable features that make it appropriate for the solution of parabolic partial differential equations. One of them is that it allows nonuniform $\xi$ and $\eta$ spacings by second-order accuracy. Because the box scheme is a common method for solving parabolic differential equations, only a brief outline is provided here. First of all, the parabolic differential equation of order $n$ is reduced to a system of $n$ first-order equations. These equations were approximated by difference equations, using central differences at each nodal point. The ensuing algebraic equations, which show a block tridiagonal structure, can be solved easily.

The numerical calculations concerning Eq. (31) were performed with the help of a uniform grid in the $\xi$ and $\eta$ directions. Numerical runs showed that approximately 50 points in the $\eta$ direction and approximately 400 points in the axial direction $(0 \leq \xi \leq 1.6)$ guaranteed sufficient accuracy.

Figure 2 shows the perturbation velocity $\tilde{u}_{1}=\partial \psi_{1} / \partial \eta$ calculated from Eq. (40), compared with the numerical solution of Eq. (31). It can be seen that the simple five-term expansion, according to Eq. (40), approximates the numerical solution quite well. Only for greater values of the axial coordinate, the deviation from the numerical calculation is more pronounced. However, for $\xi \rightarrow \infty$, the perturbation velocity $\tilde{u}_{1}$, calculated from Eq. (40), and the numerically calculated distributions are identical.

Inserting the expressions for the streamfunctions $\psi_{0}$ and $\psi_{1}$, given by Eqs. (27) and (40), respectively, into the energy Eq. (25) for the quantity $\theta_{1}$, the following partial differential equation for $\theta_{1}$ can be obtained by using Eq. (30) for $\theta_{0}$ :

$$
\begin{array}{r}
\frac{3}{2}\left(1-\eta^{2}\right) \frac{\partial \theta_{1}}{\partial \xi}-\frac{1}{\operatorname{Pr}} \frac{\partial^{2} \theta_{1}}{\partial \eta^{2}}=\frac{\partial \psi_{1}}{\partial \xi} \sum_{n=1}^{\infty} A_{n} F_{n}^{\prime}(\eta) \exp \left(-\frac{2}{3} \frac{\xi}{\operatorname{Pr}} \lambda_{n}^{2}\right) \\
+\frac{\partial \psi_{1}}{\partial \eta} \sum_{n=1}^{\infty} \frac{2}{3 \operatorname{Pr}} \lambda_{n}^{2} A_{n} F_{n}(\eta) \exp \left(-\frac{2}{3} \frac{\xi}{\operatorname{Pr}} \lambda_{n}^{2}\right)
\end{array}
$$

The boundary conditions belonging to Eq. (41) are 


$$
\begin{gathered}
\xi=0: \theta_{1}=0 \\
\eta=0: \frac{\partial \theta_{1}}{\partial \eta}=0 \\
\eta=1: \theta_{1}=0
\end{gathered}
$$

By ignoring all terms on the right-hand side of Eq. (41) it can be seen that the resulting homogeneous equation reduces to Eq. (28). Therefore, the solution of Eq. (41) can be assumed in the form (see, e.g., Myers, 1987)

$$
\theta_{1}=\sum_{m=1}^{\infty} B_{m}(\xi) F_{m}(\eta)
$$

Equation (43) satisfies identically the boundary conditions on $\theta_{1}$ for $\eta=0$ and $\eta=1$ because of the used eigenfunctions $F_{m}(\eta)$ according to Eq. (30). Inserting the expression for $\theta_{1}$, given by Eq. (43), into Eq. (41) and using the orthogonality relation for the eigenfunctions $F_{m}(\eta)$, results in a linear differential equation for the functions $B_{m}(\xi)$

$$
\frac{d B_{m}(\xi)}{d \xi}=-\frac{2}{3} \frac{\lambda_{m}^{2}}{\operatorname{Pr}} B_{m}(\xi)+\frac{2}{3} \frac{1}{K_{m}} \int_{0}^{1} F_{m}(\eta) Q(\xi, \eta) d \eta
$$

with the abbreviations

$$
\begin{gathered}
K_{m}=\int_{0}^{1}\left(1-\eta^{2}\right) F_{m}^{2}(\eta) d \eta \\
Q(\xi, \eta)=\frac{\partial \psi_{1}}{\partial \xi} \sum_{n=1}^{\infty} A_{n} F_{n}^{\prime}(\eta) \exp \left(-\frac{2}{3} \frac{\xi}{\operatorname{Pr}} \lambda_{n}^{2}\right) \\
+\frac{\partial \psi_{1}}{\partial \eta} \sum_{n=1}^{\infty} \frac{2}{3 \operatorname{Pr}} \lambda_{n}^{2} A_{n} F_{n}(\eta) \exp \left(-\frac{2}{3} \frac{\xi}{\operatorname{Pr}} \lambda_{n}^{2}\right)
\end{gathered}
$$

The resulting boundary condition for $B_{m}(\xi)$ was obtained from $\mathrm{Eq}$. (42)

$$
\xi=0: B_{m}=0
$$

Equation (44) can be solved analytically. After some routine but rather longish algebra the following expression for $B_{m}(\xi)$ was obtained:

$$
\begin{aligned}
B_{m}(\xi)= & \frac{2}{3} \frac{1}{K_{m}} \sum_{n=1}^{\infty} \int_{0}^{1} A_{n} F_{n}^{\prime}(\eta) F_{m}(\eta)\left(\exp \left(-\frac{2}{3} \frac{\xi}{\operatorname{Pr}} \lambda_{n}^{2}\right)\right. \\
& \left.\times D_{1}(\xi, \eta)-\exp \left(-\frac{2}{3} \frac{\xi}{\operatorname{Pr}} \lambda_{m}^{2}\right) D_{1}(0, \eta)\right) d \eta \\
+ & \frac{4}{9} \frac{1}{K_{m} \operatorname{Pr}} \sum_{n=1}^{\infty} \int_{0}^{1} \lambda_{n}^{2} A_{n} F_{n}(\eta) F_{m}(\eta)\left(\exp \left(-\frac{2}{3} \frac{\xi}{\operatorname{Pr}} \lambda_{n}^{2}\right)\right. \\
& \left.\times D_{2}(\xi, \eta)-\exp \left(-\frac{2}{3} \frac{\xi}{\operatorname{Pr}} \lambda_{m}^{2}\right) D_{2}(0, \eta)\right) d \eta \\
+\frac{4}{9} \frac{1}{K_{m} \operatorname{Pr}} \sum_{n=1, m \neq n}^{\infty} \int_{0}^{1} \frac{\lambda_{n}^{2} A_{n} F_{n}(\eta) F_{m}(\eta)}{3 \operatorname{Pr}}\left(\bar{\lambda}_{m}^{2}-\bar{\lambda}_{n}^{2}\right) & \exp \left(-\frac{2}{3} \frac{\xi}{\operatorname{Pr}} \lambda_{n}^{2}\right) \\
& \left.\times D_{3}(\xi, \eta)-\exp \left(-\frac{2}{3} \frac{\xi}{\operatorname{Pr}} \lambda_{m}^{2}\right) D_{3}(0, \eta)\right) d \eta \\
+ & \frac{4}{9} \frac{\xi}{K_{m} \operatorname{Pr}} \exp \left(-\frac{2}{3} \frac{\xi}{\operatorname{Pr}} \lambda_{m}^{2}\right) \int_{0}^{1} \lambda_{m}^{2} A_{m} F_{m}^{2}(\eta) D_{3}(\xi, \eta) d \eta
\end{aligned}
$$

In Eq. (47) the functions $D_{1}(\xi, \eta), D_{2}(\xi, \eta)$ and $D_{3}(\xi, \eta)$ were used. These functions are given by

$D_{1}(\xi, \eta)=\frac{b A(\eta)}{\frac{2}{3 \operatorname{Pr}}\left(\bar{\lambda}_{m}^{2}-\bar{\lambda}_{n}^{2}\right)+b} \exp (b \xi)$

$$
\begin{aligned}
& +\frac{1}{\left(\frac{2}{3 \operatorname{Pr}}\left(\tilde{\lambda}_{m}^{2}-\tilde{\lambda}_{n}^{2}\right)+a\right)^{2}+\omega^{2}} \exp (a \xi) \\
& +\left[( B ( \eta ) \frac { a ^ { 2 } } { \omega } + C ( \eta ) \frac { a } { \omega } - B ( \eta ) \omega ) \left(\left(\frac{2}{3 \operatorname{Pr}}\left(\tilde{\lambda}_{m}^{2}-\bar{\lambda}_{n}^{2}\right)+a\right)\right.\right. \\
& \times \sin (\omega \xi)-\omega \cos (\omega \xi))(2 B(\eta) a+C(\eta)) \\
& \left.\times\left(\left(\frac{2}{3 \operatorname{Pr}}\left(\tilde{\lambda}_{m}^{2}-\bar{\lambda}_{n}^{2}\right)+a\right) \cos (\omega \xi)+\omega \sin (\omega \xi)\right)\right] \\
& D_{2}(\xi, \eta)=\frac{A^{\prime}(\eta)}{\frac{2}{3 \operatorname{Pr}}\left(\tilde{\lambda}_{m}^{2}-\tilde{\lambda}_{n}^{2}\right)+b} \exp (b \xi) \\
& +\frac{1}{\left(\frac{2}{3 \operatorname{Pr}}\left(\bar{\lambda}_{m}^{2}-\bar{\lambda}_{n}^{2}\right)+a\right)^{2}+\omega^{2}} \exp (a \xi) \\
& {\left[B^{\prime}(\eta)\left(\left(\frac{2}{3 \operatorname{Pr}}\left(\bar{\lambda}_{m}^{2}-\tilde{\lambda}_{n}^{2}\right)+a\right) \cos (\omega \xi)+\omega \sin (\omega \xi)\right)+\left(\frac{C^{\prime}(\eta)}{\omega}\right.\right.} \\
& \left.\left.+B^{\prime}(\eta) \frac{a}{\omega}\right)\left(\left(\frac{2}{3 \operatorname{Pr}}\left(\tilde{\lambda}_{m}^{2}-\tilde{\lambda}_{n}^{2}\right)+a\right) \sin (\omega \xi)-\cos (\omega \xi)\right)\right] \\
& D_{3}(\xi, \eta)=\frac{9}{168}-\frac{99}{280} \eta^{2}+\frac{9}{24} \eta^{4}-\frac{9}{120} \eta^{6}
\end{aligned}
$$

Inserting Eq. (47) into Eq. (43) results in the solution $\theta_{1}$, which satisfies the given boundary conditions according to Eq. (42).

The Temperature Distribution in the Solid Region. With the boundary conditions (9), the temperature distribution in the solid phase is easily calculated from Eq. (8). The temperature distribution adopts the following form:

$$
\theta_{S}=\frac{1-\tilde{y}}{1-\tilde{\delta}}
$$

The temperature gradient at the solid-liquid interface is given in dimensionless form by

$$
\frac{\partial \theta_{S}}{\partial \tilde{y}}=-\frac{1}{1-\tilde{\delta}}
$$

The Solid-Liquid Interface. After solving Eqs. (21)-(26), the temperature gradient in the fluid at the solid-liquid interface is known. Therefore, the development of the ice layer thickness can be calculated from Eq. (10) at every axial position. Introducing Eq. (50) into Eq. (10) results in

$$
\left(\frac{\partial \widetilde{\delta}}{\partial \tau}\right)_{\bar{\gamma}}=\frac{1}{\tilde{\delta}-1}-\left.\frac{1}{B \tilde{\delta}} \frac{\partial \theta}{\partial \eta}\right|_{\eta=1}
$$

in which terms of the order $\left(\partial^{2} \tilde{\delta} / \partial \xi^{2}\right)$ were neglected.

The temperature gradient in the fluid at the solid-liquid interface, which appears in Eq. (51), is given by

$$
\left.\frac{\partial \theta}{\partial \eta}\right|_{\eta=1}=\left.\frac{\partial \theta_{0}}{\partial \eta}\right|_{\eta=1}+\left.\frac{1}{\tilde{\delta}} \frac{\partial \tilde{\delta}}{\partial \xi} \frac{\partial \theta_{1}}{\partial \eta}\right|_{\eta=1}+\ldots
$$

with the known functions $\partial \theta_{0} /\left.\partial \eta\right|_{\eta=1}$ and $\partial \theta_{1} /\left.\partial \eta\right|_{\eta=1}$, which can be calculated from Eqs. (30) and (43) by differentiating the expressions with respect to $\eta$ and evaluating the resulting functions at $\eta=1$. It should be noted here that $\partial \theta_{0} /\left.\partial \theta_{\eta}\right|_{\eta=1}$ 


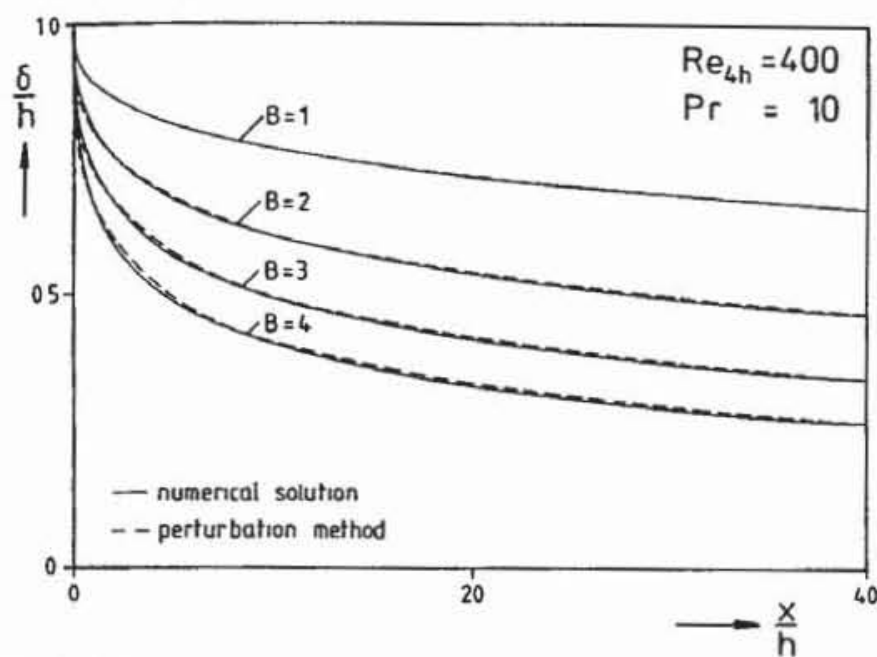

Fig. 3 Steady-state ice layer thickness as a function of $x / h$ for $R e_{4 n}=400$, $\operatorname{Pr}=10$, and various $B$

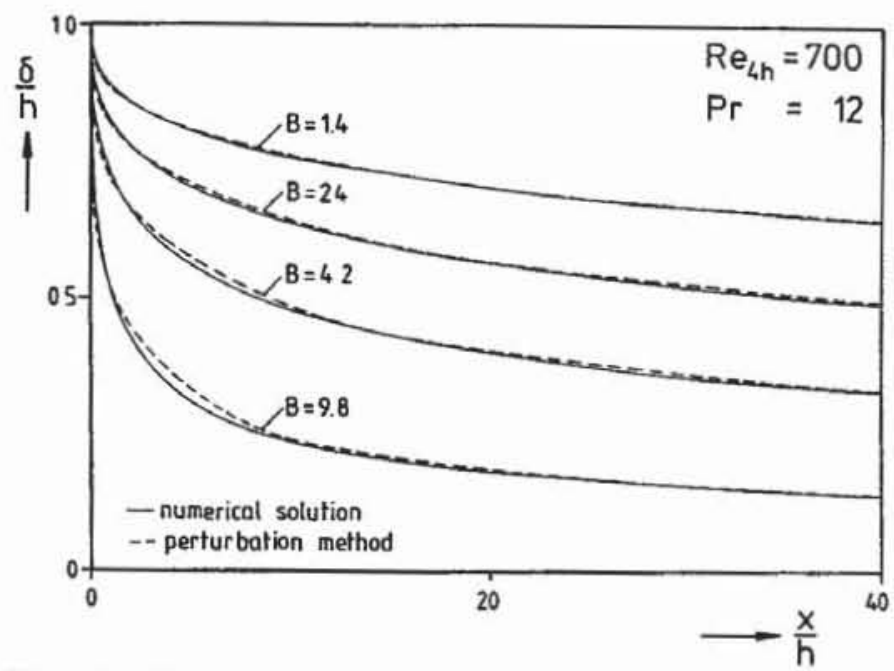

Fig. 4 Steady-state ice layer thickness as a function of $x / h$ for $R e_{4 h}=700$, $\operatorname{Pr}=12$, and various $B$

and $\partial \theta_{1} /\left.\partial \eta\right|_{\eta=1}$ are only functions of $\xi$ and Pr. They do not depend explicitly on $\tilde{\delta}$ or $\operatorname{Re}_{h}$.

Equation (51) is a nonlinear integrodifferential equation, because the derivation of $\tilde{\delta}$ with time has to be taken at constant $\tilde{x}$ and the temperature gradient in the fluid at the solid-liquid interface is a function of the integral coordinate $\xi$, defined in Eq. (13).

By ignoring terms of the order $(\partial \tilde{\delta} / \partial \tilde{x})$ in Eqs. (51) and (52), Weigand and Beer (1992) were able to derive a simple approximative solution for the transient development of the frozen layer. They obtained the free channel height $\tilde{\delta}$ as an implicit function of $\xi / \mathrm{Re}_{h} / \mathrm{Pr}$ for a given value of the freezing parameter $B$. The distribution of $\delta$ for the steady-state solution and for a parabolic entrance velocity profile was identical to the solution given by Lee and Zerkle (1969).

Taking terms into account of the order $(\partial \tilde{\delta} / \partial \hat{x})$ in Eqs. (51) and (52), Eq. (51) must be integrated numerically. This was done by using the Runge-Kutta method. Approximately 200 grid points in the axial direction were used for the calculation of the development of the frozen crust at the cooled channel walls $(0 \leq \tilde{x} \leq 20)$. The calculations were performed with a time step $\Delta \tau$ of approximately $10^{-4}$. It must be pointed out that the calculation of $\tilde{\delta}$ at each time step involves an iteration, because the perturbation quantity $(1 / \bar{\delta})(\partial \widetilde{\delta} / \partial \xi)$ for the temperature gradient at the solid-liquid interface appears in Eq. (52) and, therefore, Eqs. (51) to (52) are coupled. However, by performing some numerical calculations, it could be shown that the perturbation quantity $(1 / \bar{\delta})(\partial \tilde{\delta} / \partial \xi)$, appearing in Eq. (52), could be approximated with good accuracy by taking the

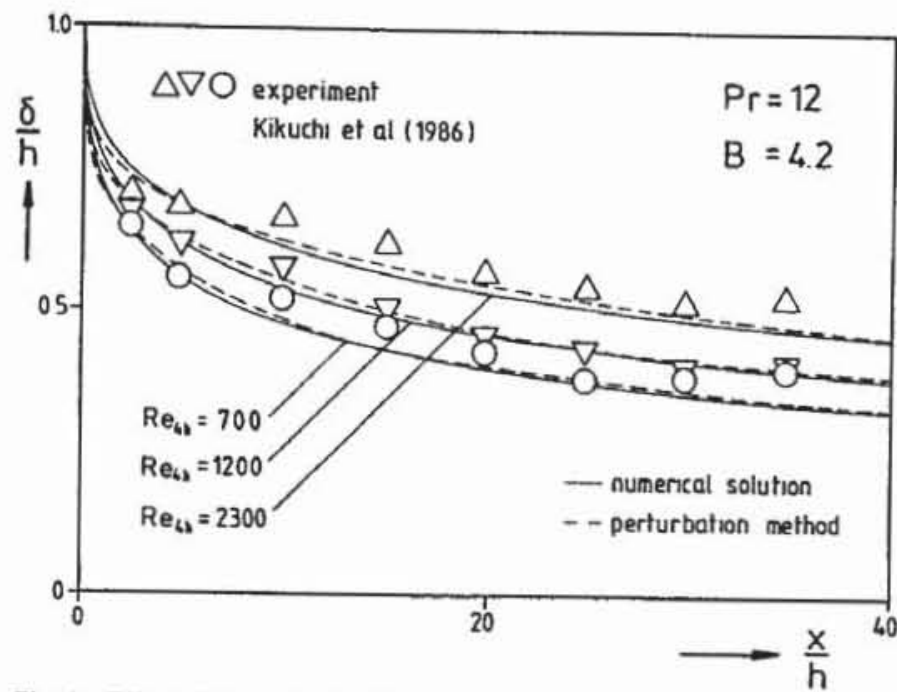

Fig.5 Effect of Reynolds number on the axial distribution of $\bar{\delta}$ for $\mathrm{Pr}=12$ and $B=4.2$

zero-order solution of Weigand and Beer (1992). This makes superfluous the iteration for $\bar{\delta}$ at a fixed value of time. The resulting error is negligible (the ice layers obtained with the two methods coincide). The steady-state solution was reached, if

$$
\left|\frac{\partial \tilde{\delta}}{\partial \tau}\right|_{\Omega}<10^{-4} ; \quad \tilde{x}=\frac{L}{h}
$$

was satisified at the end of the cooled test section.

\section{Results and Discussion}

Steady-State Freezing Fronts. The validity of the given perturbation analysis will be checked for the steady-state solution. This yields an upper limit of error, as $(1 / \delta)(\partial \tilde{\delta} / \partial \xi)$ will reach its maximum for steady-state conditions. In Figs. 3 and 4 the steady-state ice layer thickness is plotted as a function of the dimensionless downstream coordinate $x / h$. It can be observed that the ice layer thickness increases with growing values of $B$ for a given Reynolds number. The two figures elucidate the deviation of the presented perturbation solution from the numerical calculation. The numerical solution was obtained by solving Eqs. (1)-(6) with a finite-difference scheme (Weigand and Beer, 1991). It can be seen that the perturbation solution is in good agreement with the numerical calculations, also for high values of the cooling parameter $B$, as it is shown in Fig. 4 for $B=9.8$.

Figure 5 elucidates the effect of increasing Reynolds number on the axial distribution of $\bar{\delta}$ for $B=4.2$ and $\mathrm{Pr}=12$, compared with experimental data of Kikuchi et al. (1986). It is obvious that the ice layer thickness decreases with an increasing Reynolds number. This is due to the increasing heat flux from the liquid to the solid-liquid interface for growing values of $\mathrm{Re}_{4 h}$. The calculated results agree well with measurements of Kikuchi et al. (1986). It can also be observed that the deviation between the numerically calculated ice layers and the perturbation solution increases with growing Reynolds number, because the perturbation quantity is proportional to $\mathrm{Re}_{4 h}$. Therefore, the perturbation solution will approximate the numerical solution closer for smaller values of $(1 / \delta)(\partial \delta / \partial \xi)$.

In case of the experimental results for $\mathrm{Re}_{4 h}=2300$, plotted in Fig. 5, the flow was still laminar. This is because of the acceleration of the flow due to converging ice layers, which tends to stabilize the laminar boundary layer and shifts the transitional Reynolds number to higher values.

The accuracy of the given perturbation analysis can be checked more precisely by comparing the axial velocity distribution given by Eqs. (27) and (40) with numerically calcu- 


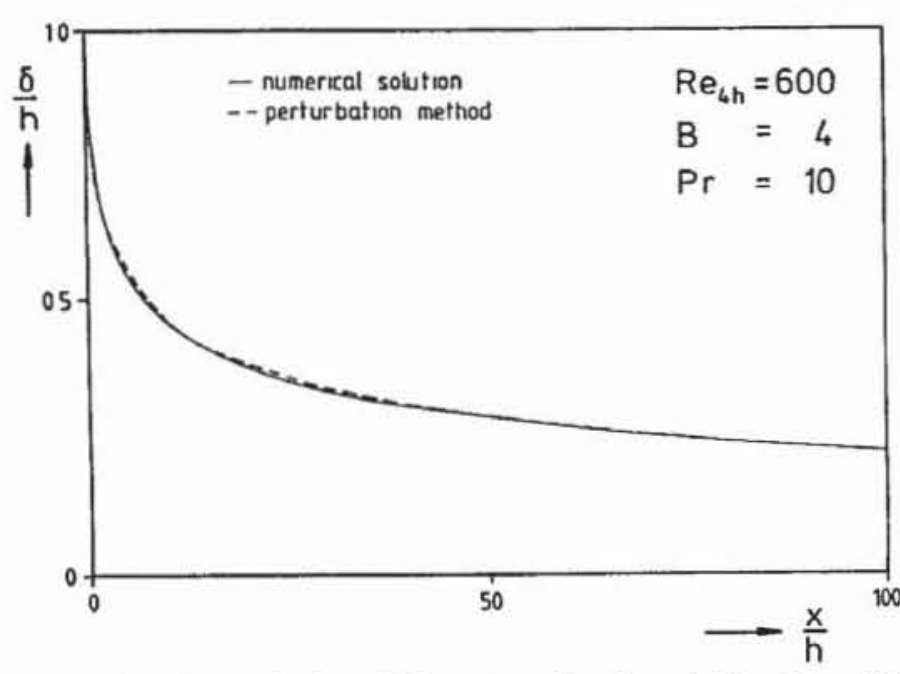

Fig. 6 Steady.state ice layer thickness as a function of $x / h$ for $R e_{4 h}=400$, $\operatorname{Pr}=10$, and $B=4$

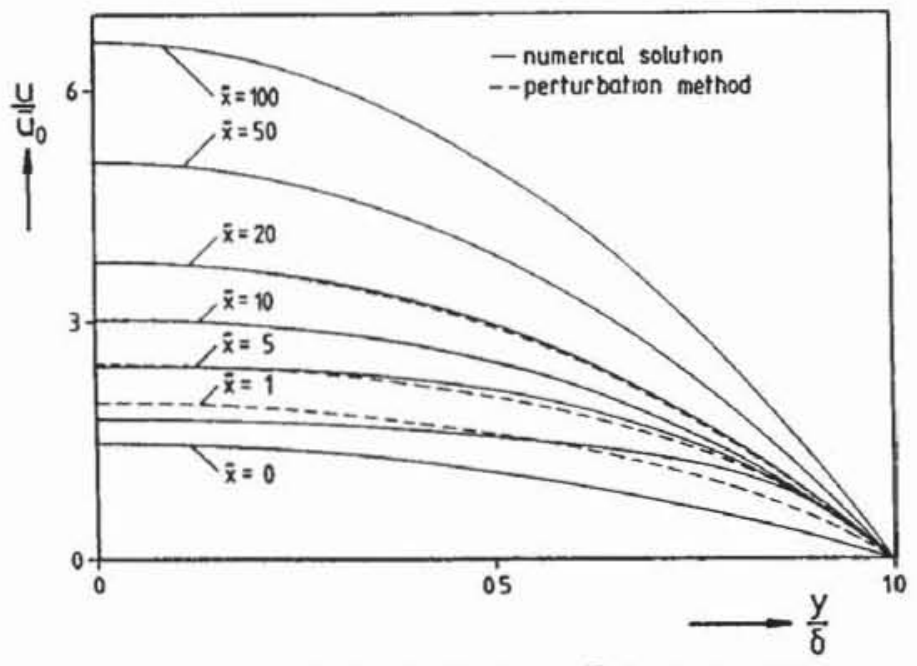

Fig. 7 Axlal velocity distributions $u \bar{u}_{0}$ for various $x / h$

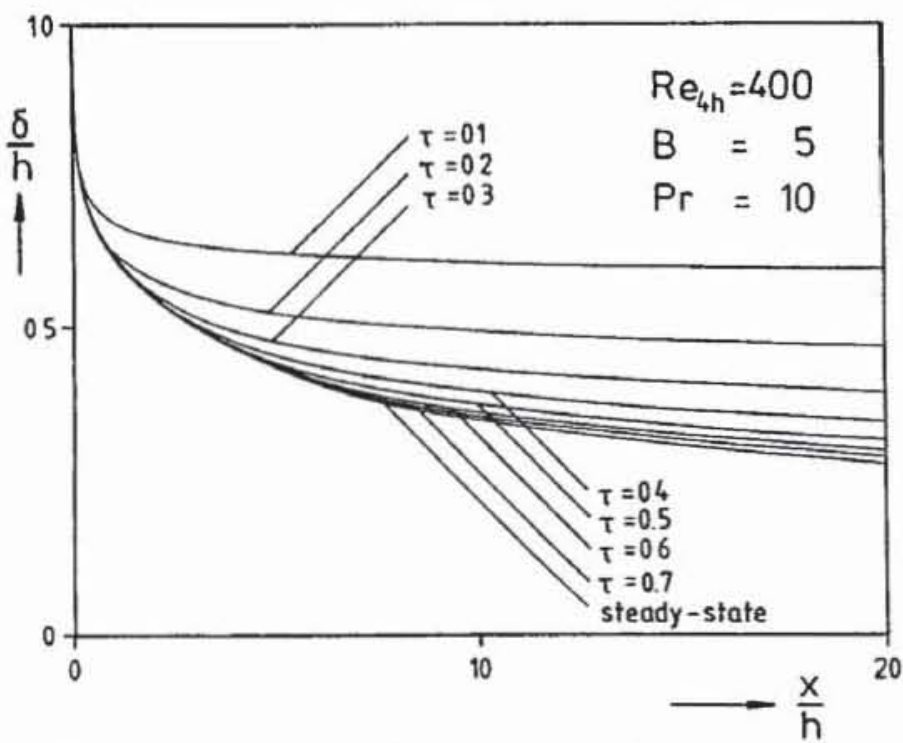

Fig. 8 Transient development of the freezing front as a function of $x / h$

lated values. Figure 6 shows a steady-state ice layer for $\mathrm{Re}_{4 h}=600, \operatorname{Pr}=12$. It can be observed that the numerical solution for $\bar{\delta}$ and the results obtained by the perturbation analysis nearly coincide.

Figure 7 illustrates the development of the axial velocity profile for the ice layer shown in Fig. 6. The axial velocity is

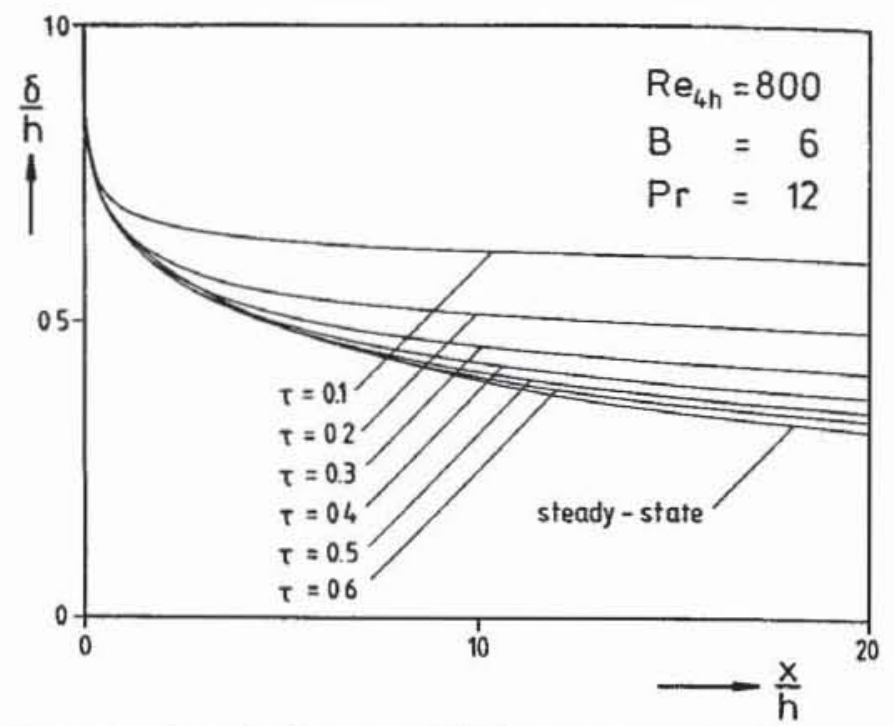

Fig. 9 Transient development of the freezing front as a function of $x / h$

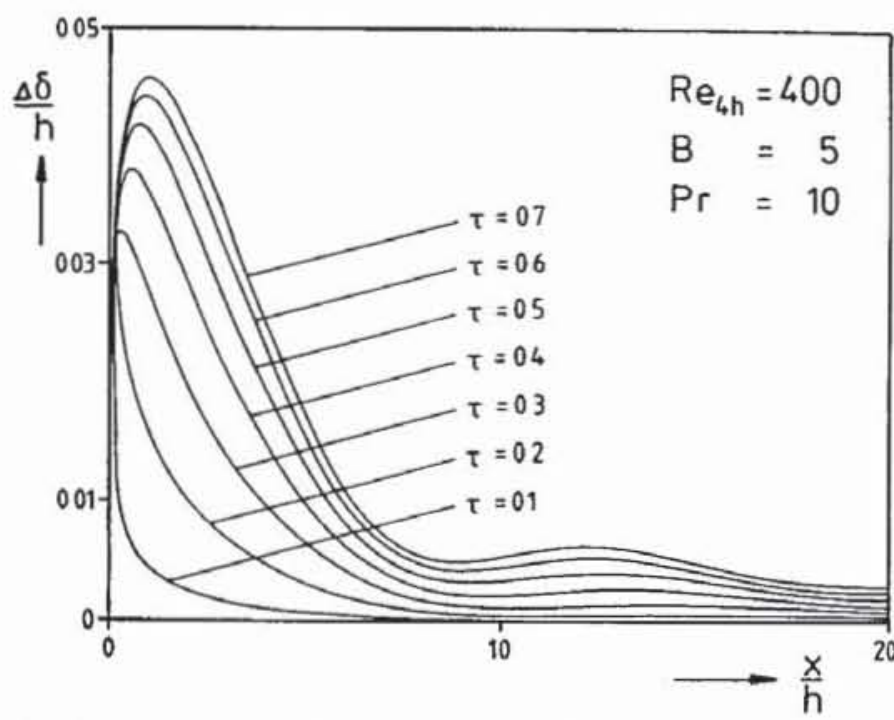

Fig. 10 Development of the absolute deviation between the perturbation solution and the approximation according to Weigand and Beer (1992) as a function of $x / h$

scaled with the axial mean velocity $\bar{u}_{0}$ at the entrance of the chill region. The profiles elucidate the effect of acceleration due to converging ice layers for various axial positions. It can be seen that the profiles are flattened for small values of the axial coordinate, while they approach a nearly parabolic shape for large values of the axial coordinate. The profiles obtained by the perturbation analysis are in good agreement with the numerical calculations, except for $\tilde{x}=1$, where the deviation between the two solutions is more pronounced. This can be attributed to the sharply increasing ice layer thickness near the entrance of the test section. Consequently, the term $\partial \tilde{\delta} / \partial \tilde{x}$ adopts higher values in this region and the linearization of the conservation equations given by Eq. (19) may lead to some error. Nevertheless, the agreement between the numerical calculation and the perturbation solution is very good for $\bar{x}>5$.

Transient Development of the Freezing Fronts. Figures 8 and 9 illustrate the time-dependent development of the freezing fronts at the channel walls for different values of the cooling parameter $B$. In the early stage of the freezing process the ice layer thickness remains approximately constant over the channel length, except for the near entrance region, as can be seen from Fig. 8 for $\tau=0.1$. This is evidenced by Eq. (51). If one excludes small values of the axial coordinate $\tilde{x}$, the first term on the right-hand side of Eq. (51) dominates the second term. 
This means that for small values of $\tau$ the growth of the frozen layer at the channel walls is dominated by heat conduction in the thin ice layer.

Because there exists no numerical solution concerning the quasi-steady development of the ice layer in a planar channel, the given results can only be compared with the approximate solution of Weigand and Beer (1992). Figure 10 shows the distribution of the absolute deviation between the perturbation solution and the approximation during the transient development of the ice layer shown in Fig. 8. It is evident that the maximum deviation is obtained for steady-state conditions, because the solution of Weigand and Beer (1992) coincides with the approximation of Lee and Zerkle (1969) for the stationary case. Moreover it can be seen that the deviation between the two solutions is maximum for small values of $x / h$. This can be easily understood if one recognizes that the quantity $\partial \tilde{\delta} / \partial \tilde{x}$ reaches its maximum for low values of the axial coordinate. Because the effect of this term on the distribution of the ice layer thickness was ignored in the analysis of Lee and Zerkle (1969) and Weigand and Beer (1992), the maximum deviation must be in this region. However, the deviation $\Delta \tilde{\delta}$ between the two solutions is smaller than 0.05 . Therefore, it can be stated that the solution given by Weigand and Beer (1992) approximates the time-dependent development of the freezing fronts in a cooled parallel plate channel relatively well.

\section{Conclusions}

A quite flexible method has been developed for calculating the velocity and temperature distributions in a planar channel with arbitrarily shaped walls under the assumptions that the perturbation quantity $(1 / \tilde{\delta})(\partial \tilde{\delta} / \partial \xi)$ is sufficiently small. The applicability of the method was provided by calculating the transient development of the ice layers in a parallel plate channel for quasi-steady conditions. By comparing the obtained steady-state solutions with the numerical calculations of Weigand and Beer (1991) and the experiments of Kikuchi et al. (1986), it was demonstrated that the perturbation solution yields sufficient accuracy for a wide range of Reynolds numbers and cooling parameters $B$.

It could be shown that the transformed Navier-Stokes equa- tions and the energy equation, for the quasi-steady conditions and written in $\xi, \eta$ coordinates, contain only powers and derivatives of $(1 / \delta)(\partial \tilde{\delta} / \partial \xi)$, but not explicitly $\tilde{\delta}^{n}$. Therefore, application of this method to the complete conservation equations for quasi-steady conditions is straightforward.

\section{References}

Andrews, L. C., and Shivamoggi, B. K., 1988, Integral Transforms for Engineers and Applied Mathematicians, Macmillan, New York.

Bennon, W. D., and Incropera, F. P., 1988, "Developing Laminar Mixed Convection With Solidification in a Vertical Channel," ASME Journal of Heat Transfer, Vol. 110, pp. 410-415.

Bilenas, J. A., and Jiji, L. M., 1970, "Numerical Solution of a Nonlinear Free Boundary Problem of Axisymmetric Fluid Flow in Tubes With Solidification," Proceedings, 4th International Heat Transfer Conference, Vol. 1, pp. $1-11$

Cebeci, T., and Chang, K. C., 1978, "A General Method for Calculating Momentum and Heat Transfer in Laminar and Turbulent Duct Flows," Numerical Heat Transfer, Vol. 1, pp. 39-68.

Cebeci, T., and Bradshaw, P., 1984, Physical and Computational Aspects of Convective Heat Transfer, Springer, New York.

Cervantes, J., Trevino, C., and Méndez, F., 1990, “An Asymptotic Analysis for the Transient Freezing and Laminar Flow in a Circular Pipe," Proceedings, 9th International Heat Transfer Conference, Vol. 4, pp. 321-326.

Chida, K., 1983, "Heat Transfer in Steady Laminar Pipe Flow With Liquid Solidification," Heat Transfer: Japanese Research, Vol. 81, pp. 81-94.

Kikuchi, Y., Shigemasa, Y., Oe, A., and Ogata, T., 1986, "Steady-State Freezing of Liquids in Laminar Flow Between Two Parallel Plates," Journal of Nuclear Science and Technology, Vol. 23, pp. 979-991.

Lee, D. G., and Zerkle, R. D., 1969, "The Effect of Liquid Solidification in a Parallel-Plate Channel Upon Laminar-Flow Heat Transfer and Pressure Drop," ASME Journal of Heat Transfer, Vol. 91, pp. 583-585.

Myers, G. E., 1987, Analytical Methods in Conduction Heat Transfer, Genium Publishing Corporation, Schenectady, NY.

Özişik, M. N., and Mulligan, J. C., 1969, "Transient Freezing of Liquids in Forced Flow Inside Circular Tubes," ASME Journal of Heat Transfer, Vol. 26, pp. 233-240.

Shah, R. K., and London, A. L., 1978, "Laminar Flow Forced Convection in Ducts," Advances in Heat Transfer, Academic Press, New York.

Weigand, B., and Beer, H., 1991, "Heat Transfer and Solidification of a Laminar Liquid Flow in a Cooled Parallel Plate Channel: The Stationary Case," Wärme- und Stoffubertragung, Vol. 26, pp. 233-240.

Weigand, B., and Beer, H., 1992, "Transient Freezing of Liquids in Forced Laminar Flow Inside a Parallel Plate Channel," Wärme- und Stoffübertragung. Vol. 27, pp. 77-84.

Zerkle, R. D., and Sunderland, J. E., 1968, "The Effect of Liquid Solidification in a Tube Upon Laminar-Flow Heat Transfer and Pressure Drop," ASME JOURnAl OF Heat Transfer, Vol. 90, pp. 183-190. 